\title{
Collaboration and development of radio-astronomy in Australasia and the South-Pacific region: New Zealand perspectives
}

\author{
Sergei A. Gulyaev and Tim J. Natusch \\ Centre for Radiophysics and Space Research, Auckland University of Technology, Private Bag \\ 92006, Auckland 1142, New Zealand \\ email: Sergei.Gulyaev@aut.ac.nz; Tim.Natusch@aut.ac.nz
}

\begin{abstract}
As a result of collective efforts of an Australian-New Zealand VLBI team, the first New Zealand VLBI system was developed, and a series of test observations between New Zealand and Australia conducted. The equipment and techniques used to conduct New Zealand's first VLBI observations are discussed and results of work in Australia and New Zealand to obtain fringes and the image of the source (PKS1921-231) are presented. The road map for New Zealand radio-astronomy as well as New Zealand involvement in the SKA is discussed.
\end{abstract}

Keywords. Radio-astronomy, VLBI, SKA

\section{Radio-astronomy and VLBI in New Zealand}

Radio-astronomy in New Zealand has links stretching back to the work of Elizabeth Alexander on solar emissions in the 1940s, and John Bolton and Gordon Stanleys mobile cliff interferometer. This latter instrument was used in New Zealand in 1947 to obtain rising and setting records of various sources. It was the first direct collaboration between New Zealand and Australia in this field. Measurements made near Sydney and Auckland determined the positions of several strong radio sources for the first time. This allowed their optical counterparts to be identified as supernova remnants and external galaxies and not "radio-stars" as previously had been assumed. A number of New Zealand pioneers, Gordon Stanley and Bruce Slee and more recently, New Zealanders Dick Manchester (ATNF) and Peter Napier (VLA) are among world famous radio-astronomers.

Current work in radio-astronomy is centred on the newly formed Centre for Radiophysics and Space Research (CRSR) located at Auckland University of Technology (AUT). AUT has undertaken the task of developing VLBI facilities in New Zealand. This work is being conducted in collaboration with Swinburne University of Technology, Melbourne and with the support of the Australia National Telescope Facility and the University of Tasmania.

A grant of $\$ N Z 300000$ was obtained from the New Zealand Ministry of Economic Development (MED) to support this work. The MED sees VLBI activity as a potential user of the Advanced Network, which in turn forms a vital component of the Governments Digital Strategy. A grant application for $\$ 5 \mathrm{M}$ for establishment of New Zealand Radio Telescope National Facility (RTNF) was recently submitted to the New Zealand Government. It is proposed to install an integrated radio-astronomical and geodetic VLBI station in the North Island and upgrade an existing old 11-m Earth station in Southland into a small radio-telescope. The main investment required is one modern 12-16 metre radio telescope, and two hydrogen atomic clocks - the standard of time and frequency 


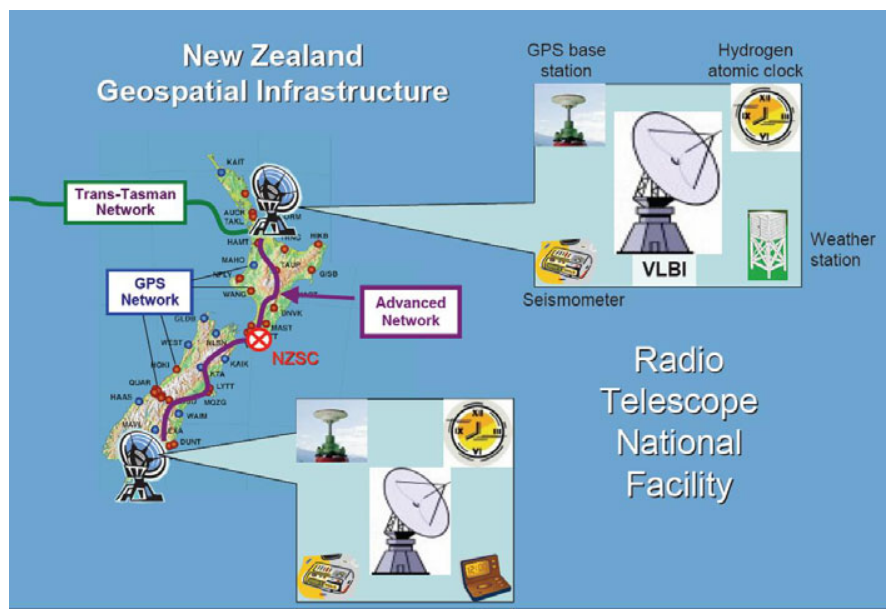

Figure 1. The concept of the Radio Telescope National Facility as a part of New Zealand geospatial and ICT infrastructure.

essential for VLBI. The proposed RTNF, along with the existing geodetic (GPS) network, Advanced Research Network (broadband) and New Zealand Supercomputer will comprise a world class national geospatial infrastructure (figure 1).

\section{Regional collaboration and development}

Radio telescopes in the Asia-Pacific region form a natural network for VLBI observations, similar to the very successful networks in North America (Network Users Group) and Europe (European VLBI Network). New Zealands VLBI facility, which we are developing since 2005, has the potential to strengthen the Asian-Pacific VLBI network and its role in astronomy, geodesy and geoscience (figure 2). It will positively influence regional and international activities in geoscience and geodesy that advance New Zealand's national interests.

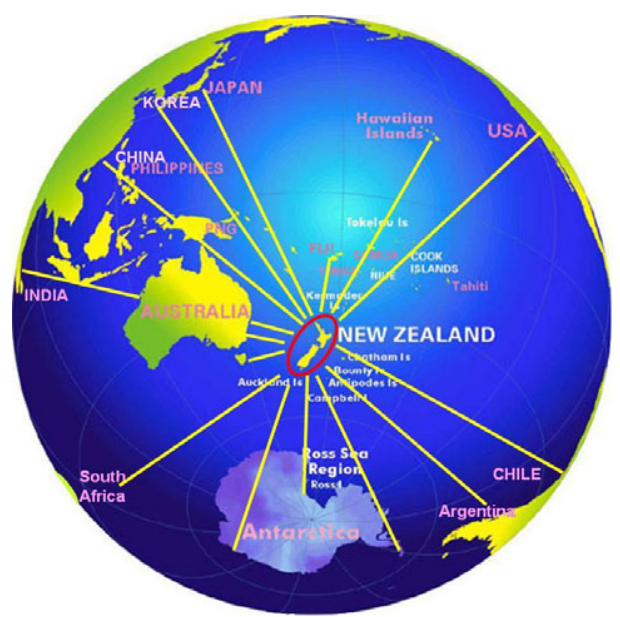

Figure 2. New Zealand's unique geographic location allowing VLBI with six continents and a number of countries in the South-Pacific region. 


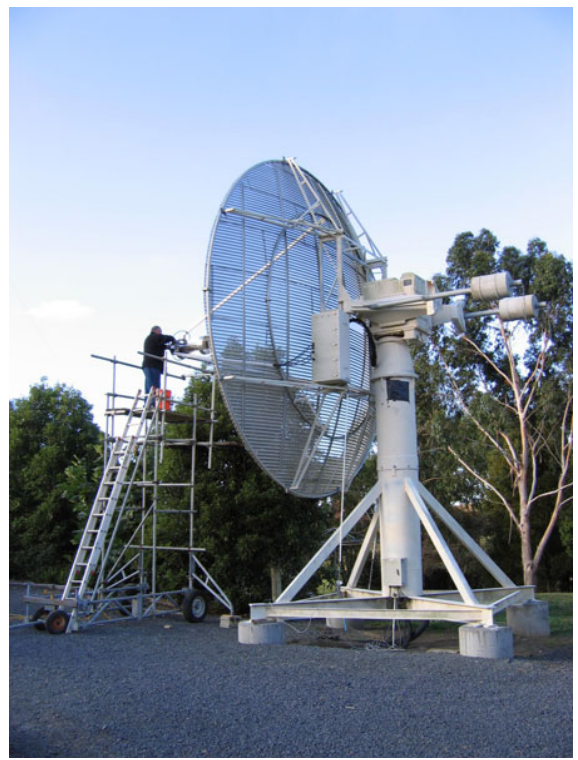

Figure 3. 6-m radio telescope (Auckland) used in the first Trans-Tasman (New Zealand-Australia) VLBI observations in 2005.

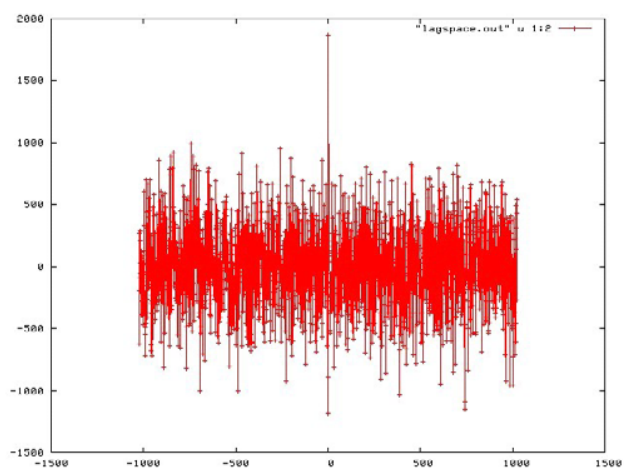

Figure 4. The first VLBI fringe obtained from New Zealand-Australia test in 2005.

A self-contained radio-astronomy system for VLBI, including a $1.658 \mathrm{GHz}$ (centre frequency), $16 \mathrm{MHz}$ bandwidth RF system (feed and downconversion system locked to a Rubidium maser and GPS clock), an 8-bit sampler with digitization system, and a diskbased recording system built around a commodity PC was developed in New Zealand Centre for Radiophysics and Space Research (Gulyaev, Natusch et al. 2005). This was designed as a portable system for use on different radio telescopes, since the one thing that New Zealand lacks at the moment is ready access to a large collecting area, fully steerable antenna.

A number of Trans-Tasman tests has been conducted in 2005-2006 between the CRSR system installed on a 6-m dish located in Auckland (figure 3) and the Australia Telescope Compact Array in Narrabri, Australia. This work has been successful, with fringes located from the recorded data (figure 4) and a high resolution image of the quasar PKS1921231 synthesized (figure 5). New Zealand has demonstrated the capacity to contribute to modern radio-astronomical and VLBI research (Gulyaev, Natusch et al. 2006). 


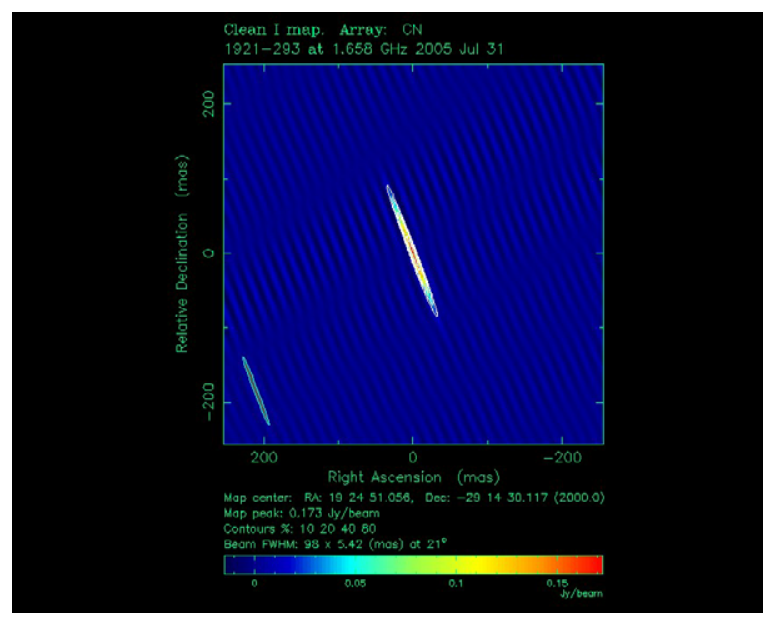

Figure 5. An image of PSK1921-231 synthesized from data obtained from New Zealand-Australia VLBI test in 2005.

Experiments were recently conducted with Kashima Radio Observatory (Japan); new tests are planned with Korea and Fiji. Plans have been made to build a new 16-m antenna in New Zealands North Island and to upgrade an 11-m dish in the South Island (see figure 1).

\section{New Zealand and the SKA}

New Zealand's geographic location makes it a natural place for possible extension of the Australian SKA from $3000 \mathrm{~km}$ to almost $6000 \mathrm{~km}$, significantly increasing the maximum spatial resolution of the SKA telescope. Four trial sites with excellent radio environments, favourable natural conditions, and well developed infrastructure and communications have been selected as potential SKA sites. One of them (Warkworth, North Island) is protected as a radio quiet zone.

\section{Acknowledgements}

We would like to thank our VLBI colleagues from Swinburne University of Technology, Australia Telescope National Facility, University of Tasmania, and Kashima Space Research Centre.

\section{References}

Gulyaev, S., Natusch, T., Addis, B., Tingay, S. \& Deller, A. 2005, Southern Stars 44, 12.

Gulyaev, S., Natusch, T., Tingay, S., Deller, A., West, C., Ellingsen, S., McCulloch, P., Reid, B., Baynes, E. \& Tzioumis, T. 2006, ATNF News 58, 8. 